\title{
Colloid-stabilized emulsions: behaviour as the interfacial tension is reduced
}

\author{
P S Clegg ${ }^{1,2}$, E M Herzig ${ }^{1,2}$, A B Schofield ${ }^{1}$, T S Horozov ${ }^{3}$, B P Binks ${ }^{3}$, \\ M E Cates ${ }^{1}$ and W C K Poon ${ }^{1,2}$ \\ ${ }^{1}$ SUPA, School of Physics, University of Edinburgh, Edinburgh, EH9 3JZ, UK \\ 2 COSMIC, University of Edinburgh, Edinburgh, EH9 3JZ, UK \\ ${ }^{3}$ Surfactant and Colloid Group, Department of Chemistry, University of Hull, \\ Hull HU6 7RX, UK
}

Received 3 October 2005

Published 28 October 2005

Online at stacks.iop.org/JPhysCM/17/S3433

\begin{abstract}
We present confocal microscopy studies of novel particle-stabilized emulsions. The novelty arises because the immiscible fluids have an accessible upper critical solution temperature. The emulsions have been created by beginning with particles dispersed in the single-fluid phase. On cooling, regions of the minority phase nucleate. While coarsening, these nuclei become coated with particles due to the associated reduction in interfacial energy. The resulting emulsion is arrested, and the particle-coated interfaces have intriguing properties. Having made use of the binary-fluid phase diagram to create the emulsion we then make use of it to study the properties of the interfaces. As the emulsion is re-heated toward the single-fluid phase the interfacial tension falls and the volume of the dispersed phase drops. Crumpling, fracture or coalescence can follow. The results show that the elasticity of the interfaces has a controlling influence over the emulsion behaviour.
\end{abstract}

\section{Introduction}

Recent studies have begun to examine the mechanism by which colloidal particles can emulsify immiscible liquids. One strand of this research has focused on different oils emulsified in water and has used silica particles as the prototype colloid [1]. If the colloid is partly wetted by both fluids then there is a large energy gain if the colloid resides on the interface between the two liquids. The benefit arises because the particle removes a region of the shared interface between the two fluids. The simplicity of the mechanism and the stability of the resulting emulsions makes this a very promising technology.

Numerous pairs of immiscible liquids will begin to mix when heated or cooled [2]. The case of liquids with an upper critical solution temperature (UCST), which mix on warming, is shown in figure 1(a). As the mixed state is approached the compositions of the two liquids change steadily and the interfacial tension between them decreases. This effect gives novel 

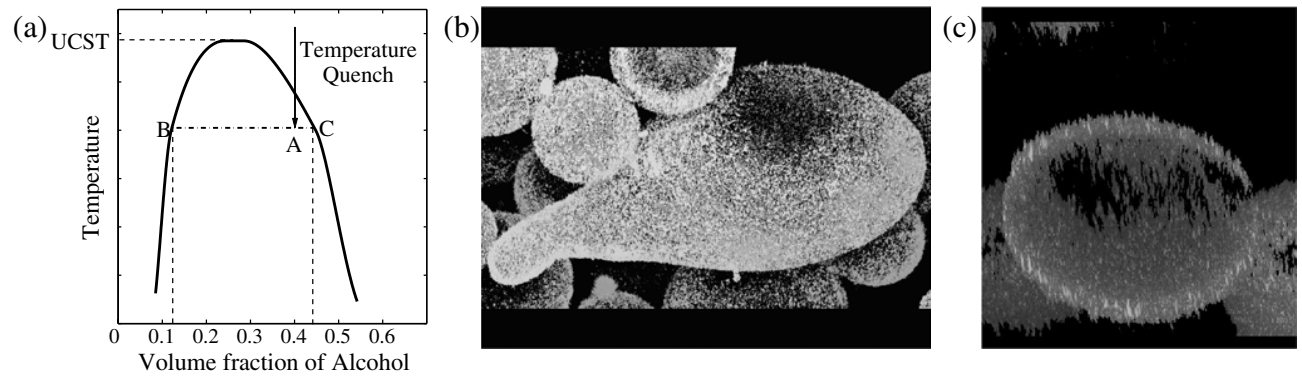

Figure 1. (a) A schematic alcohol-oil phase diagram showing the quench route used here. The bold line is the coexistence curve and the UCST is the upper critical solution temperature. A sample quenched to A will nucleate droplets with composition B coexisting with a continuous background of composition C. ((b), (c)) A hexane-in-methanol emulsion is stabilized by silica particles following a slow shallow quench into the demixed region. These images are a projection of colloidal stacks and they show that there is no systematic distortion due to gravity (pulling them up). The vertical dimension is $200 \mu \mathrm{m}$.

opportunities for studying particle-stabilized emulsions. It makes it possible for us to observe the behaviour of the droplets as the balance of volumes of the two fluids changes continuously. In addition the effect of vanishing interfacial tension on the colloid layer can be observed directly.

In this paper we study alcohol-oil emulsions stabilized by large silica colloids. The particles themselves are $\sim 0.5 \mu \mathrm{m}$ diameter Stöber silica [3] with modified surface chemistry. The surface has been partially silanized to give a wetting angle close to $90^{\circ}$ for the alcohol-oilsilica interface [4]. In the systems studied here we have used methanol-hexane, methanolheptane and ethanol-dodecane as the alcohol-oil combinations. For these pairs the upper critical points are in a convenient temperature range $\left(12-53^{\circ} \mathrm{C}\right)$. The liquid compositions (generally 2:3 alcohol:oil by volume) were away from the critical composition (see the quench route in figure $1(\mathrm{a})$ ) and particle volume fractions of $\sim 1 \%$ were used. The force keeping a large colloidal particle on a liquid-liquid interface is surprisingly large. Close to the neutral wetting condition, a $\sim 0.5 \mu \mathrm{m}$ particle can change the interfacial energy by $\sim 10^{4} k_{\mathrm{B}} T$. We used a Nikon confocal microscope with a Biorad Ar-ion laser to examine the samples. Rendering three-dimensional images was carried out using the ImageJ software package [5].

Our studies were performed by emulsifying the liquids using the silica particles and observing the changes as the liquids were warmed toward miscibility. We found evidence that the particle-stabilized interfaces are solid. The solid behaviour of particles trapped at flat liquid-liquid interfaces has previously been studied in isolation [6, 8]. Furthermore, in emulsion science and technology the case of protein-stabilized interfaces has similarities with that of the emulsion considered here [9]. Globular proteins often begin to bond with each other on liquid-liquid interfaces and this strengthens the resulting emulsion. Here we show the behaviour of densely laden interfaces as the interfacial tension falls.

\section{Behaviour of droplets as the interfacial tension changes}

\subsection{Creation by nucleation}

The emulsification route used here is novel. Many of our emulsions have been created by beginning with the miscible liquid pair and the colloids dispersed in this single-fluid phase. The interfaces and concomitant interfacial tension only appear as the sample is quenched into the two-phase region, figure 1(a). Emulsions form on cooling as particles are swept up by the 


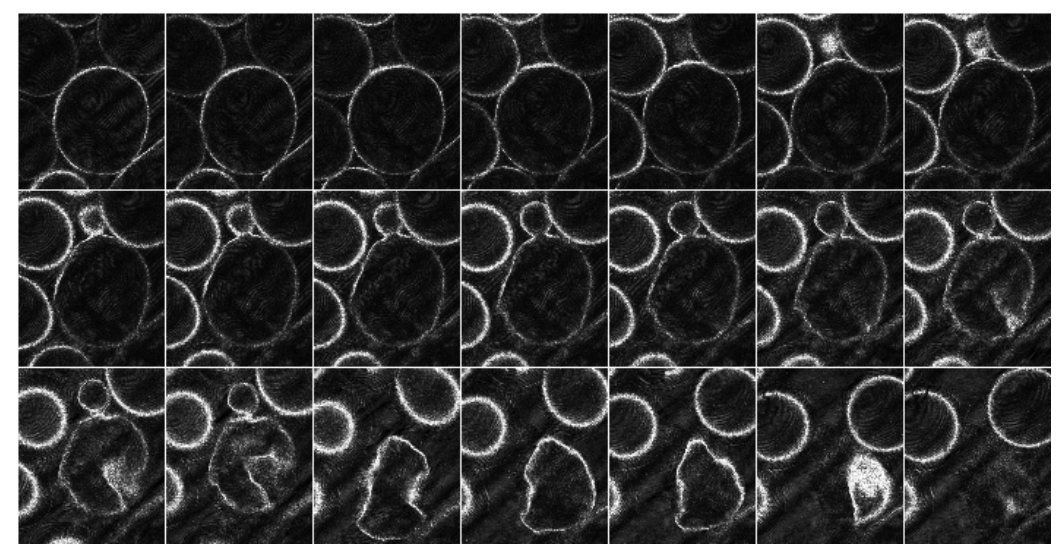

Figure 2. Images of a heptane-in-methanol droplet deflating. Beginning in the top left-hand corner, there is one frame every $60 \mathrm{~s}$ with each $2{ }^{\circ} \mathrm{C}$ higher in temperature than the previous one. The bulk demixing temperature is just before the end of this sequence. The images are at a depth of $40 \mu \mathrm{m}$ from the surface of the holder and are roughly $300 \mu \mathrm{m}$ on an edge.

interfaces of the expanding droplets. The layer of colloids at the interface eventually becomes very dense. The droplets stop growing and are stable for many hours; figures 1(b) and (c). The final length scale of the structure is controlled by the geometry of surface coverage. The droplet diameter is $\xi \sim n R / \phi_{v}$ where $R$ is the colloid radius, $\phi_{v}$ is their volume fraction and $n$ is the number of layers of particles. In the samples studied here the interfaces are covered by half-a-dozen layers of particles and this is consistent with the observation that $\xi \sim 150 \mu \mathrm{m}$.

A comparison between the particle-free case and the emulsified droplets created here is instructive. For alcohol-oil mixtures undergoing demixing, gravity will eventually cause the droplets to cream to the top of the sample and coalesce. In pure alcohol-oil mixtures this leads to macroscopic phase separation. The response to creaming is evidently strongly modified by the colloids. The droplets still rise due to gravity; however they maintain their integrity due to the particle-stabilized interfaces. The coalescence mechanism for droplets emulsified by a conventional surfactant has been studied for many years [10]. The role of fluctuations in the interfacial coverage and that of the equilibrium between surfactant in solution and on the interface have been shown to be important. These effects are all associated with the surfactant layer being fluid. Here the colloidal particles are very densely packed and it seems unlikely that regions of exposed interface will appear by fluctuation alone. The dense interface will strongly oppose the formation of necks between two droplets. Hence, as we observe, the colloid-stabilized emulsion is robust to coalescence.

\subsection{Destruction on warming}

To demonstrate that the fluid phase separation has given rigidity to the particle-laden interfaces we have studied the behaviour as both the interfacial tension and the balance of volumes are varied. We achieve this by slowly warming our particle-stabilized emulsions into the mixed regime. Figure 2 shows a series of frames including a central droplet which becomes deflated. This is a very clear change to the behaviour shown in figures 1(b) and (c), for example. In figure 2 the interfaces are not stable and are rapidly changing in shape. An important point to note is that most of the surrounding droplets are not crumpling and this suggests that the integrity of the droplet surface for this individual droplet has been lost. (The slow creaming of the droplets in these frames indicates that none of them are stuck to the sample holder.) 


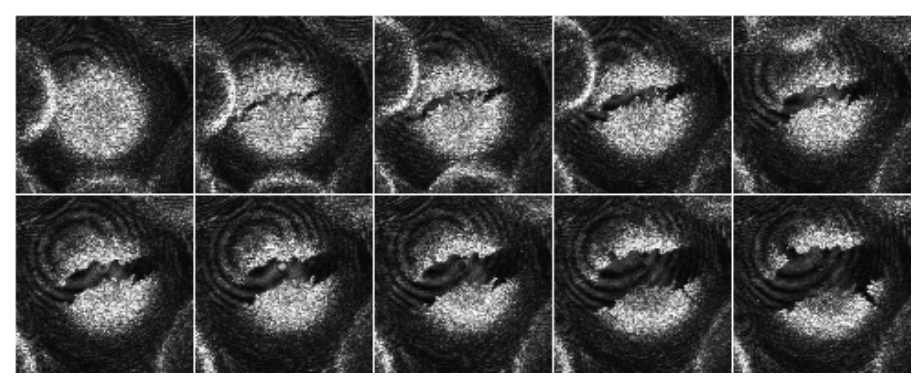

Figure 3. Images of a heptane-in-methanol droplet cracking. Beginning in the top left-hand corner, there is one frame every $30 \mathrm{~s}$ with each $1{ }^{\circ} \mathrm{C}$ higher in temperature than the previous one. The bulk demixing temperature is in the middle of this sequence. The images are at a depth of $40 \mu \mathrm{m}$ from the surface of the holder and are roughly $120 \mu \mathrm{m}$ on an edge.

The puncture in the particle skin allows the minority fluid phase to leave the shell and mix with the continuous phase. A structure with a high bending modulus will tend to maintain smoothly curved surfaces when it deflates. This phenomenon is well known from studies of blood cells and some types of vesicle [11]. If the internal pressure is reduced the structure of these can transform into oblate shapes including a biconcave structure or prolate shapes including dumb-bells. This is not what we see on warming this sample. Instead, the shape we observe is puckered and creased. This behaviour indicates that our particle-laden interfaces are dominated by the energy cost of stretching the interface. Similar crumpling behaviour is observed when a water-in-crude-oil droplet is deflated using a micropipette [12].

Other researchers have studied the conditions leading to crumpling of flat particle layers at liquid-liquid interfaces using a Langmuir trough [6, 7]. It is found that a buckling transition occurs when the inter-particle repulsion exactly balances the interfacial tension cost of expanding the region between particles [6]. It seems likely that a related transition is occurring here as we slowly reduce the interfacial tension and internal volume.

We suggested above that puncturing of the droplet surface may have led to the observed deflation. We have associated other observations with droplets retaining their integrity to higher temperatures. In figure 3 it is shown that droplets with a complete coverage of particles will eventually shatter. This effect is often seen in the thin side walls of emulsion droplets; however it is most visually dramatic to observe the fracture in the end face of a droplet. The cracking reveals the solidity of these particle layers. The layers are sufficiently rigid that they fracture rather than distort.

\subsection{Coalescence and stability}

As the emulsions are heated toward miscibility coalescence becomes increasingly common. Figure 4(a) shows a smaller droplet coalescing with its larger neighbour. The combined interface becomes smooth and round within minutes. This contrasts strongly with behaviour at low temperatures. Evidently in some cases the layer of colloids is becoming sufficiently flexible and fluid for conventional coalescence behaviour to occur. An interface of negative Gaussian curvature is stable for a few tens of seconds (figure 4(a) central panel).

Such coalescence obviously can also occur during cooling. Figures 4(b) and (c) show two permanently distorted droplets. In both cases there are regions of the interface with negative Gaussian curvature. The peanut and trefoil droplets may well have fused while the surfaces were fluid without having time to rearrange before the bending and Young's moduli became too high. It can be concluded that the large elastic moduli make it impossible for the droplet 

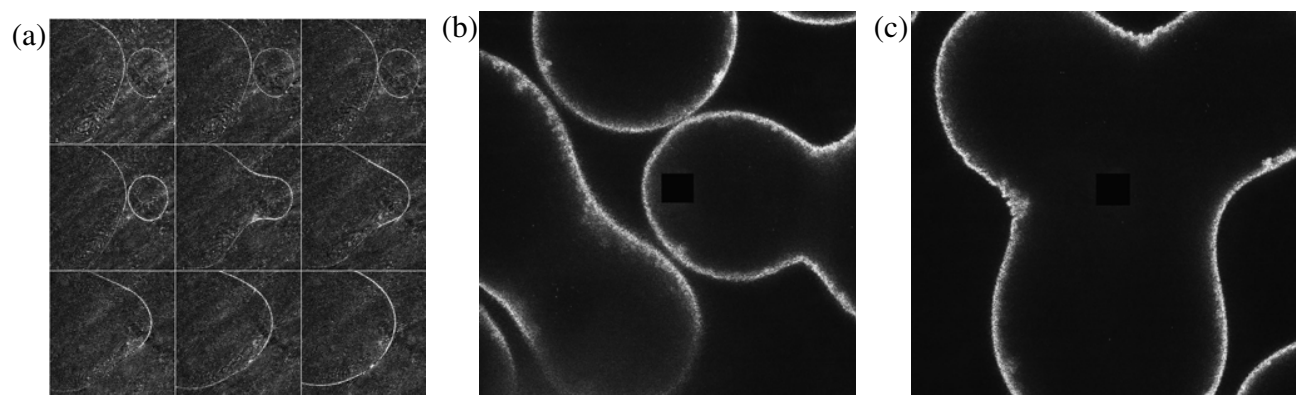

Figure 4. (a) Coalescence in progress in a dodecane-in-ethanol emulsion. Beginning in the top left-hand corner, there is one frame (500 $\mu \mathrm{m}$ across) every $30 \mathrm{~s}$ with $1{ }^{\circ} \mathrm{C}$ between them. ((b), (c)) Distorted heptane-in-methanol droplets, most likely due to coalescence during cooling. The images are $200 \mu \mathrm{m}$ across and are of different regions of the same sample.

surface to change its shape. In addition the particle interfaces are slightly wrinkled at the points of sharpest curvature in figure 4(c) and this suggests that the surface is solid and the particles are unable to desorb.

We note that the existence of peanut/dumb-bell shapes (figures 4(b) and (c)) does not by itself demonstrate that the particle surfaces are solid. Similar shapes are predicted for vesicles with fluid surfaces of non-zero rigidity within both the spontaneous-curvature and bilayercoupling models $[11,13]$. Instead it is the crumpling and fracture behaviour which are the key to understanding the nature of these emulsions.

\section{Summary}

We have presented confocal microscopy images of droplet geometries in particle-stabilized emulsions. We have shown how the behaviour dramatically changes as the fluids slowly become miscible. The droplets created in our experiments have surface layers of particles that are solid and have relatively high elastic moduli. At low temperature the system is essentially arrested and can make no attempt to rearrange itself on experimentally relevant timescales.

Our results begin to reveal the conditions under which the coalescence of droplets can occur. Figures 1(b) and (c), figure 2 (upper images) and figures 4(b) and (c) show particlestabilized droplets which have been in close contact for hours. It is clear that the droplets are showing no tendency toward coalescing. By contrast, figure 4(a) shows coalescence in action. It appears that fluidity of the layers is essential before two droplets can coalesce. This is an important observation concerning why particle-stabilized monolayers have longevity. The results presented here are qualitative. We intend to follow up our observations with a study of the rheology of dense layers of silica particles at liquid-liquid interfaces [8].

The creation route for these emulsions has involved a quench from high temperature into the demixed region of the phase diagram. It is an interesting extension of this research to explore the behaviour at higher quench rates to lower temperatures. One approach would be to see how the particles respond to different separation kinetics and the resulting structures could be highly novel. These studies are under way.

\section{Acknowledgments}

We thank S U Egelhaaf and D Roux for illuminating discussions. Funding in Edinburgh was provided by the EPSRC (Grant GR/S10377/01). 


\section{References}

[1] Aveyard R, Binks B P and Clint J H 2003 Adv. Colloid Interface Sci. 100-102 503

[2] Rowlinson J S and Swinton F L 1982 Liquids and Liquid Mixtures 3rd edn (London: Butterworths)

[3] Stöber W, Fink A and Bohn E 1968 J. Colloid Interface Sci. 2662

[4] Horozov T S, Aveyard R, Clint J H and Binks B P 2003 Langmuir 192822

[5] Rasband W S 1997-2005 ImageJ (Bethesda, MD: US National Institute of Health) http://rsb.info.nih.gov/ij/

[6] Aveyard R, Clint J H, Nees D and Quirke N 2000 Langmuir 168820

[7] Vella D, Aussillous P and Mahadevan L 2004 Europhys. Lett. 68212

[8] Stancik E J, Hawkinson A L, Vermant J and Fuller G G 2004 J. Rheol. 48159

[9] Dickinson E 1999 Colloids Surf. B 15161

[10] Evans D F and Wennerström H 1999 The Colloidal Domain 2nd edn (New York: Wiley-VCH)

[11] Pamplona D C and Calladine C R 1996 J. Biomech. Eng. 118482

[12] Yeung A, Dabros T, Czarnecki J and Masliyah J 1999 Proc. R. Soc. A 4553709

[13] Seifert U, Berndl K and Lipowsky R 1991 Phys. Rev. A 441182 